\title{
Epigallocatechin-3-gallate decreases UVA-induced HPRT mutations in human skin fibroblasts accompanied by increased rates of senescence and apoptosis
}

\author{
YANG XU ${ }^{1}$, JIE ZHU ${ }^{2}$ BINGRONG ZHOU ${ }^{1}$ and DAN LUO ${ }^{1}$ \\ ${ }^{1}$ Department of Dermatology, The First Affiliated Hospital of Nanjing Medical University; \\ ${ }^{2}$ Department of Dermatology, Jiangsu Province Hospital of Traditional Chinese Medicine, Nanjing, Jiangsu, P.R. China
}

Received December 15, 2011; Accepted January 20, 2012

DOI: $10.3892 /$ etm.2012.466

\begin{abstract}
Our study was designed to determine the protective effect of epigallocatechin-3-gallate (EGCG) on cultured human skin fibroblasts (HSFs) from multiple ultraviolet A (UVA) irradiation-induced hypoxanthine-guanine phosphoribosyl transferase (HPRT) mutant colony formation and its underlying mechanisms. In our study, the mutation frequency of the HPRT gene was examined by mutagenesis assay. Cell senescence was determined by histochemical staining of senescence-associated $\beta$-galactosidase. The apoptosis rate was detected by flow cytometry. EGCG decreased the UVA-induced HPRT gene mutation frequency by $47.85 \%$. However, EGCG further increased the number of senescent cells by $38.92 \%$ and the apoptosis rate by $56.92 \%$ in HSFs. The photo-protective effect of EGCG on multiple UVA-exposed HSFs is related to a significant reduction in UVA-induced HPRT mutant cells. This may be caused by the induction of damaged cells to proceed to senescence and apoptosis.
\end{abstract}

\section{Introduction}

Exposure of the human skin to ultraviolet (UV) irradiation can cause photoaging and photo-carcinogenesis. It is well documented that UV irradiation causes oxidative attack and damage to DNA primarily by formation of photoproducts, and even initiates targeted gene mutation in cells $(1,2)$. Subsequently, the UV-damaged cell begins DNA repair or turns to apoptosis. If the injury is too severe to be repaired completely and correctly, the damaged cells may begin an initiative event of mutant colony formation followed by cancer development.

Chemoprevention of cancer is a novel and more effective means of cancer management. Natural agents are considered

Correspondence to: Professor Dan Luo, Department of Dermatology, The First Affiliated Hospital of Nanjing Medical University, Nanjing, Jiangsu, P.R. China

E-mail: daniluo2005@yahoo.com.cn

Key words: epigallocatechin-3-gallate, ultraviolet A irradiation, senescence, mutation frequency, apoptosis less toxic and more effective in controlling various human malignancies, including skin cancer (3-5). It has been proven that epigallocatechin-3-gallate (EGCG) extracted from green tea is characterized by effects of anti-oxidation, antiinflammation and immunoregulation both in vitro and in vivo (6-8). Our previous studies proved that EGCG protects human keratinocytes and Langerhans cells from UVB-induced photodamage $(8,9)$. However, the effect of EGCG on mutant colony formation of cultured human skin fibroblasts (HSFs) caused by multiple ultraviolet A (UVA) irradiation and its underlying mechanism remain unclear.

The X-chromosomal gene for hypoxanthine-guanine phosphoribosyl transferase (HPRT), first recognized by its human germinal mutations, quickly became a useful target for studies on somatic mutations in vitro and in vivo in human beings and animals. In this role, HPRT serves as a simple reporter gene. The HPRT gene locus is sensitive to irradiation and can be an index of irradiation dosage effect $(10,11)$. Previously, senescence and apoptosis have been considered to be a crucial defensive mechanism preventing damaged or abnormal cells from cancergenesis, which has been used in cancer treatment (12-16). It has been confirmed that senescence-associated $\beta$-galactosidase (SA- $\beta$-Gal) expression increases in aging individuals and serves as an aging-related biological marker (17). In this study, we investigated the effect of EGCG on the frequency of mutations of HSFs with multiple UVA irradiations for 2 weeks. We also compared the effects of EGCG on apoptosis and SA- $\beta-\mathrm{Gal}$ expression in HSFs between the intrinsic senescence group and the multiple UVA irradiation-induced senescence group, aiming to ascertain the underlying mechanism.

\section{Materials and methods}

Reagents. Dulbecco's modified Eagle's medium (DMEM; Gibco/BRL, USA); epigallocatechin-3-gallate (Sigma, USA); MTT (3-(4,5-dimethylthiazol-2-yl)-2,5-diphenyl-tetrazolium bromide) (Sigma); solar simulator (Sigma); dispase (Sigma); $\beta$-galactosidase kit (Mirus Bio Co., USA); 6-thioguanine (Sigma); 0.2\% methylene blue (Sigma); propidium iodide (PI; Molecular Probe, USA); Vidas Image and Analysis system (Zeiss, Germany) and a flow cytometer (Epics, USA) were obtained. 
Table I. Effects of different concentrations of EGCG on UVA-irradiated HSF viability.

\begin{tabular}{lccccc}
\hline A values & \multicolumn{5}{c}{ EGCG $(\mu \mathrm{g} / \mathrm{ml})$} \\
\cline { 2 - 6 } & 0 & 10 & 25 & 50 & 100 \\
\hline 0 UVA $\left(\mathrm{J} / \mathrm{cm}^{2}\right)^{\mathrm{a}}$ & $0.54 \pm 0.13$ & $0.50 \pm 0.07$ & $0.44 \pm 0.08$ & $0.47 \pm 0.06$ & $0.52 \pm 0.10$ \\
$10 \mathrm{UVA}\left(\mathrm{J} / \mathrm{cm}^{2}\right)^{\mathrm{b}}$ & $0.34 \pm 0.02$ & $0.41 \pm 0.01$ & $0.46 \pm 0.02$ & $0.47 \pm 0.01$ & $0.40 \pm 0.02$
\end{tabular}

${ }^{a}$ There was no significance in cellular viability between the control and EGCG-treated groups without UVA irradiation ( $\left.p>0.05\right) .{ }^{b}$ The cellular viability of HSFs increased stably, while EGCG concentration ranged from 10 to $50 \mu \mathrm{g} / \mathrm{ml}$ under $10 \mathrm{~J} / \mathrm{cm}^{2} \mathrm{UVA}$ irradiation ( $\mathrm{p}<0.05$ ).

Cell culture and subgroups. Human fibroblasts derived from the foreskin of young donors ( $<5$ years of age) were isolated and cultured in DMEM (Gibco/BRL) supplemented with $2 \mathrm{mM}$ glutamine (Gibco/BRL) and $10 \%$ fetal bovine serum (HyClone) at $37^{\circ} \mathrm{C}$ in $5 \% \mathrm{CO}_{2}$. In the intrinsic senescence experiment, HSFs were cultured and passaged for a total of 80 days. In the UVA irradiation-related experiment, a serum-free version of the above medium was supplied. When growth of HSFs reached the desired confluence, the cells were divided into the following subgroups: control group, EGCG group, UVA irradiation group and UVA+EGCG group. HSFs in different groups were used for the determination of senescence, HPRT gene mutation and cellular apoptosis.

Preparation and selection of EGCG solution with optimal concentration. The EGCG solution was prepared with DMEM at the concentration of $500 \mu \mathrm{g} / \mathrm{ml}$, and stored at $-20^{\circ} \mathrm{C}$. A cell suspension $(100 \mu \mathrm{l})$ of HSFs $\left(10^{5}\right.$ cells $\left./ \mathrm{ml}\right)$ was seeded in a 96-well plate. In the UVA+EGCG group, different concentrations $(0,25,50$ and $100 \mu \mathrm{g} / \mathrm{ml})$ of EGCG solution were added. After incubation for $24 \mathrm{~h}$, the cultures were irradiated with $10 \mathrm{~J} / \mathrm{cm}^{2}$ UVA. HSFs were incubated with the new medium containing EGCG for another $24 \mathrm{~h}$ and then $20 \mu 1 \mathrm{MTT}$ $(5 \mathrm{mg} / \mathrm{ml})$ was added. After another $4 \mathrm{~h}$, the supernatant was replaced by $100 \mu \mathrm{l} \mathrm{DMSO}$, and the culture was oscillated at room temperature for $15 \mathrm{~min}$. The absorbance (A value) at $490 \mathrm{~nm}$ was measured and the cell proliferation viability in each group was determined. Since the experiments related to the SA- $\beta-$ Gal and HPRT gene mutation required a relatively longer culture time, the optimal concentration of $25 \mu \mathrm{g} / \mathrm{ml}$ EGCG was chosen in the following photo-protection study according to the preliminary cell viability assay.

UV irradiation protocol. HSFs were pre-cultured with $25 \mu \mathrm{g} /$ $\mathrm{ml}$ EGCG solution for $2 \mathrm{~h}$, then irradiated with $10 \mathrm{~J} / \mathrm{cm}^{2} \mathrm{UVA}$. The accumulation course was designed for 2 weeks. After washing twice with phosphate-buffered saline (PBS), cells were irradiated with a thin cover of PBS to avoid drying and with a water bath at room temperature to avoid overheating during irradiation. The intensity of UVA $(320-400 \mathrm{~nm})$ emitted by a solar simulator (Sigma) was $4.4 \mathrm{~mW} / \mathrm{cm}^{2}$. The irradiation distance between the cultured cells and the UV source was $15 \mathrm{~cm}$ and the irradiation dosage was controlled by a radiometer equipped with a UVA-sensor. Sham-irradiated cultures were handled identically except that they were shielded with aluminum foil during the irradiation. Each treatment was conducted in triplicates and the experiments were conducted three times.

HPRT mutagenesis assay. The HPRT-mutagenesis assay was used as described previously to detect and to characterize UV-induced HPRT mutations. Exponentially growing cells were irradiated with UVA or sham when the HSF culture reached $\sim 50 \%$ confluence. After irradiation, cells were propagated for 3.5-4 population doublings (expression period), as verified by cell counts in parallel dishes, to allow the expression of the mutated HPRT gene. After being cultured for 7-14 days (expression period), the cells were transferred to 10-20 tissue culture dishes with a selective medium containing $7 \mu \mathrm{g} / \mathrm{ml} 6$-thioguanine (Sigma), at a density of $5 \times 100 \mathrm{cells} / \mathrm{cm}^{2}$. In this selective medium, only HPRT-mutated cells were able to survive and form colonies, since they are unable to metabolize 6-thioguanine to a toxic agent. After a selection period of 4-6 weeks, HPRT-mutated, 6-thioguanine-resistant cell colonies were stained with $0.2 \%$ methylene blue and counted to determine the mutation frequency. The mutation frequency was calculated as the number of mutants/the number of plated cell $\mathrm{x}$ the plating efficiency. The latter was determined by plating 100 cells/dish separately in non-selective medium at the end of the expression period.

Histochemical method for $\beta$-galactosidase detection. The semi-quantitative analysis of SA- $\beta$-Gal-positive cells was performed when the confluence of the plated HSFs reached $50 \%$. Cells were washed in PBS, fixed in $2 \%$ formaldehyde $/ 0.2 \%$ glutaraldehyde for $5 \mathrm{~min}$ (room temperature), washed again and then incubated at $37^{\circ} \mathrm{C}\left(\right.$ no $\left.\mathrm{CO}_{2}\right)$ with fresh SA- $\beta-$ Gal stain solution. The blue-colored cells were considered $\beta$-Gal-positive cells. Randomly selected 500 cells within a field under the microscope were counted. The percentage of positive cells, which represented the aging rate of the HSF cultures, was calculated. Senescence rate $=$ the number of blue colored cells/the total cell number x $100 \%$.

Cellular apoptosis detection. The HSFs were treated as above by UVA with or without EGCG. Conditioned cells were digested by $0.25 \%$ trypsin and fixed by $80 \%$ alcohol solution. The cells were washed three times and $200 \mu \mathrm{l}$ PI $(50 \mu \mathrm{g} / \mathrm{ml})$ was added into every tube. Cells were then resuspended into a single-cell suspension and left to set for $30 \mathrm{~min}$ at room temperature. Cellular apoptosis was detected by flow cytometry. 
A

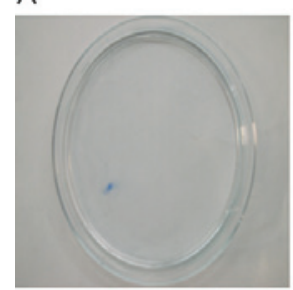

a EGCG

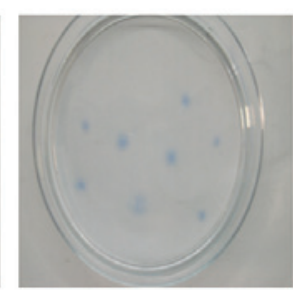

b UVA

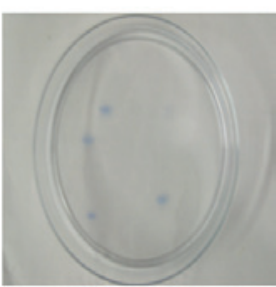

c UVA+EGCG
B

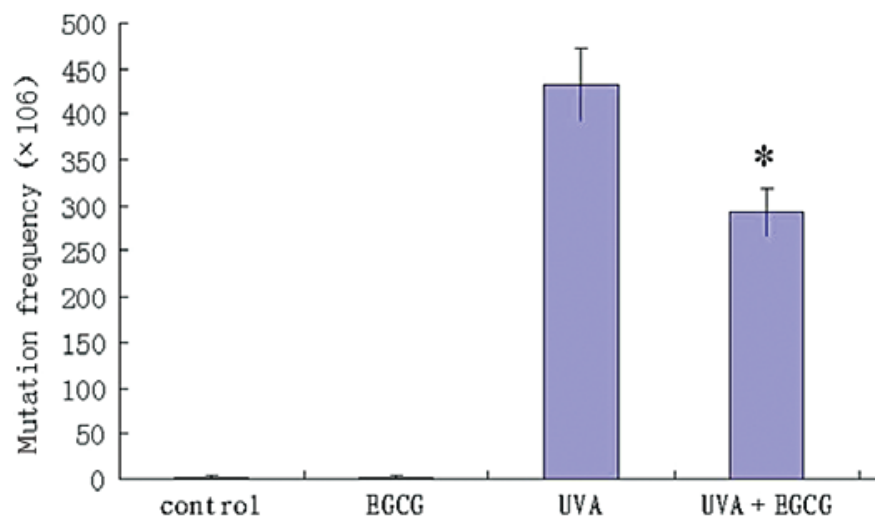

Figure 1. Effect of EGCG on UVA-irradiated cell mutation colony frequency in HSFs. (A) After the expression period, the four groups of HSF cells were cultured with $7 \mu \mathrm{g} / \mathrm{ml}$ 6-TG. After a selection period of 4-6 weeks, HPRTmutant, 6-TG-resistant cell colonies were stained with $0.2 \%$ methylene blue and counted for mutation frequency, showing that treatment of HSF with EGCG prior to UVA exposure caused a decrease in HPRT mutation $(\mathrm{a}, \mathrm{b}$ and c). (B) Statistical analysis for the data: Compared to the intrinsic mutation frequency in the control and EGCG groups, the mutation frequency in the UVA group was 241 times higher. With EGCG treatment, the mutation frequency was reduced by $32.37 \%$. Data are the means $\pm \mathrm{SD}$ of three independent experiments. ${ }^{*} \mathrm{p}<0.05$ when comparing EGCG-treated irradiated cells to irradiated cells.

Statistical analysis. SPSS 11.0 software was used to conduct the paired t-test or AVONA test. Statistical significance was established at $\mathrm{p}$-values $<0.05$.

\section{Results}

Effects of different concentrations of EGCG solution on UVA-irradiated HSF viability. Table I shows that there was no significant difference in cellular viability between the control and the EGCG-treated groups without UVA irradiation ( $p>0.05$ ), which meant that various concentrations of EGCG exhibited no cytotoxicity to the cultured HSFs. The cellular viability of the HSFs stably increased, while the EGCG concentration ranged from 10 to $50 \mu \mathrm{g} / \mathrm{ml}$ under $10 \mathrm{~J} / \mathrm{cm}^{2} \mathrm{UVA}$ irradiation $(\mathrm{p}<0.05)$. Since the experiments related to $\mathrm{SA}-\beta-\mathrm{Gal}$ and HPRT gene mutations require a relatively longer culture time, such as 80 days, for spontaneous senescence study and 2 weeks for UVR-associated experiments, $25 \mu \mathrm{g} / \mathrm{ml} \mathrm{EGCG}$ was thus chosen for further study.

Effect of EGCG on mutation colony frequency in irradiated HSF s. Different groups of HSF cells were co-cultured with 6-TG in the selective period and then stained, counted and corrected. The intrinsic mutation frequency was very low in
$\mathbf{A}$

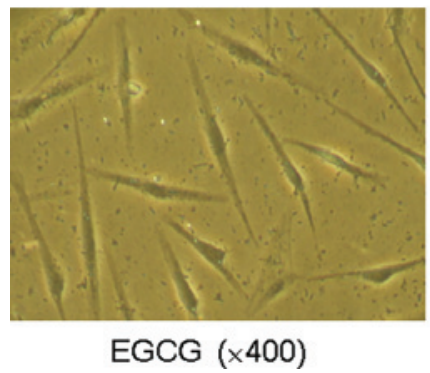

B

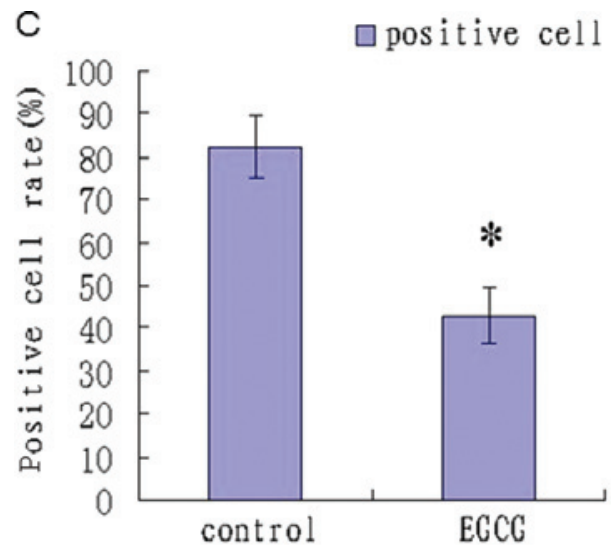

Figure 2. Effect of EGCG on the natural senescence in HSFs. After culturing and passaging HSFs for 80 days, histochemistry staining with SA- $\beta$-Gal antibody was carried out and semi-quantitative analysis for positive cells was conducted on the slide with conditioned HSFs. (A) Under microscopy, HSFs cultured with EGCG treatment grew well, displaying long shuttle or irregular shape such as fresh and young fibroblasts. With prolonged days of culture, the HSFs cultured without EGCG underwent aging and were arranged in a disordered pattern. (B) The aging HSFs appeared large and flat with many granules and vacuoles in the cytoplasm, showing an increase in the ratio of cytoplasm/nucleus. (C) Statistical analysis of positive cells and data are shown as the means \pm SD of three independent experiments. Blue/browncolored cells were counted under the microscope in order to calculate the percentage of positive cells in randomly selected 500 cells within 3 vision fields. Senescence rate $=$ the number of blue colored cells/the total cell number x $100 \%$. ${ }^{*}$ p $<0.05$ when comparing EGCG-treated cells to untreated cells.

the control group (1.8 \pm 1.6$)$ and the EGCG group (2.8 \pm 1.6$)$. The mutation frequency in the UVA group was $433.8 \pm 40.6$, almost 240 times higher than that in the control group and the simple EGCG group, respectively $(\mathrm{p}<0.001)$. Compared to simple UVA irradiation, the mutation number in the EGCG+UVA group was much lower $(293.4 \pm 27.2)$ and was reduced by $47.85 \%$ (Fig. 1).

Effect of EGCG on the intrinsic senescence in HSFs. Naturally, HSFs were passed for more than 20 passages during 80 culture days, and histochemistry was used to detect SA- $\beta$ Gal-positive cells. Under microscopy, young fibroblasts grew well, displaying a long shuttle or irregular shape (Fig. 2B). In contrast, with a prolonged culture period, the aging cells arranged in a disordered pattern were large and flat, with more cytoplasm and a higher ratio of cellular cytoplasm/ cellular nucleus. Many granules and vacuoles appeared in the cytoplasm, which was hardly observed in the HSFs treated with $25 \mu \mathrm{g} / \mathrm{ml}$ EGCG, but obvious in the aging cells without EGCG treatment (Fig. 2B), which also implied some type of cell natural senescence and functional decline. The number of 
A

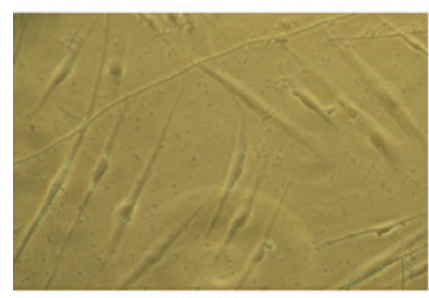

control

C

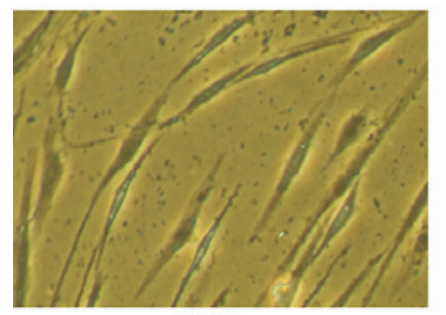

UVA
B

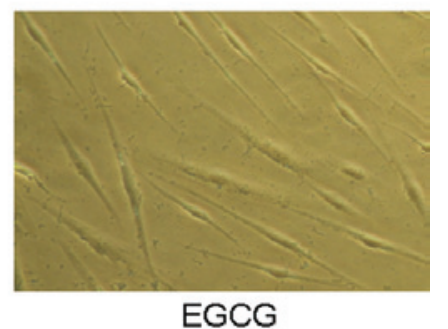

D

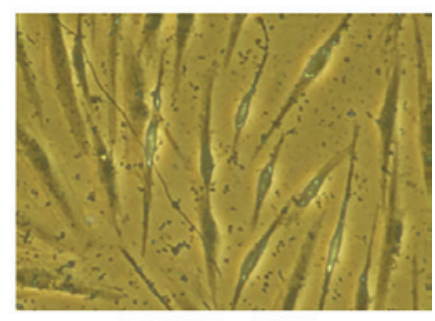

EGCG +UVA

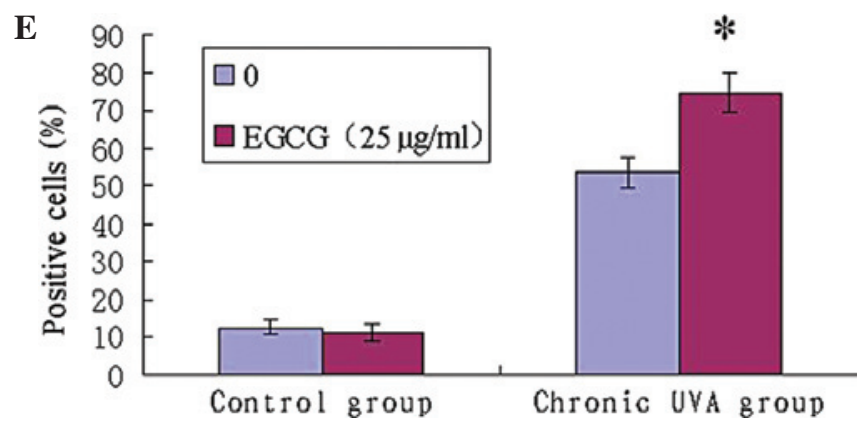

Figure 3. Effect of EGCG on UVA-irradiated cell senescence in HSFs. HSFs were managed for senescence experiment under EGCG treatment and/or UVA irradiation. (A-C) The number of $\beta$-Gal-positive stained cells showed an increasing trend in the multiple UVA group compared to the control and single EGCG-treatment groups. (D) Treatment of HSFs with EGCG prior to UVA exposure caused a further increase in the senescence rate (EGCG+UVA group). (E) Statistical analysis of positive cells; data are the means $\pm \mathrm{SD}$ of three independent experiments and the significant difference was confirmed. * $\mathrm{p}<0.05$ when comparing EGCG-treated irradiated cells to irradiated cells.

SA- $\beta$-Gal-positive cells was quite different between the two groups $(82.30 \pm 7.21$ in the control group vs. $43.12 \pm 6.48$ in the EGCG-treated group) (Fig. 2C). The senescence rate of the HSFs cultured with $25 \mu \mathrm{g} / \mathrm{ml}$ EGCG was reduced by $47.60 \%$.

Effect of EGCG on UVA-irradiated cell senescence in HSFs. After the HSFs were pre-cultured with $25 \mu \mathrm{g} / \mathrm{ml} \mathrm{EGCG} \mathrm{and/or}$ UVA irradiation for 2 weeks, cell morphology and SA- $\beta$-Gal positively-stained senescent cells were assessed in the cultures (Fig. 3A-D). Images of the UVA+EGCG group showed several large and flat cells. By histochemical staining, less SA- $\beta$-Galpositive cells were observed in the control group $(12.71 \pm 2.10)$ and the EGCG group (11.46 \pm 2.27$)$. The positive senescent cells in the multiple UVA group showed an approximately three times higher value than the control and EGCG groups (53.62 \pm 4.01$)$, and the senescence rate was also increased by $38.92 \%$ in the EGCG-multiple UVA group compared to the UVA group (74.49 \pm 5.03$)$. Statistical analysis confirmed that there was statistical significance between the UVA and EGCG+UVA groups (Fig. 3E), which implied some promo-

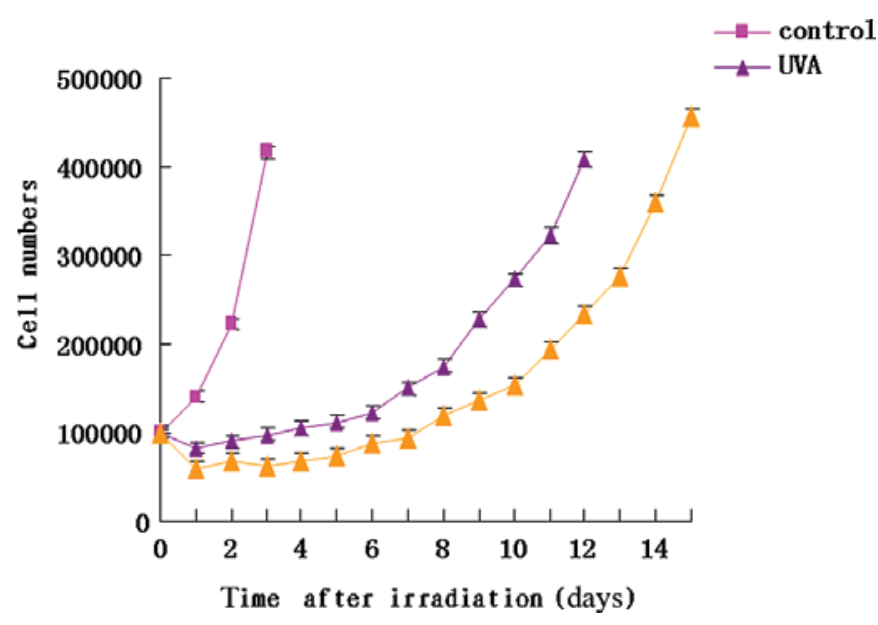

Figure 4. Cell growth curve during the expression period after EGCG and/ or multiple UVA irradiation. Compared to the control group (squared mark), the peak time of HSF proliferation was longer (11 and 15 days, respectively) in the UVA group (triangle mark in purple color) and the EGCG+UVA group (triangle mark in orange color). UVA caused a decrease in cell proliferation rate, which was further reduced by treating HSF with EGCG prior to UVA exposure.

tive effect of EGCG on cell senescence, while combined with multiple UVA irradiation.

Effect of EGCG on UVA-induced cell growth curve during the expression period. After 2 weeks of UVA irradiation, the HSFs required several days to allow for the expression of the mutant phenotype prior to replanting the mutant cells. Compared to the control group, the expression period was longer in the multiple UV irradiation group as the cells proliferated slowly and it required 1 week to enter into rapid growth period. Furthermore, the HSF cells proliferated much more slowly after the cultures were intervened by EGCG treatment. The period for the HSF cells in the UVA+EGCG group to reach a growth peak was 14 days (Fig. 4).

Effect of EGCG on UVA-induced cell apoptosis and cell cycle in HSFs. The apoptosis rates of the different groups of HSF cells were also analyzed after 2 weeks of EGCG treatment with or without UVA. There was no noticeable difference in the peak of apoptosis in the control $(0.15 \pm 0.06 \%)$ and the EGCG groups $(0.50 \pm 0.11 \%)(p>0.05)$. Compared to these two groups, the apoptosis rate was apparently higher in the chronic UVA irradiation group $(20.06 \pm 1.14 \%)$, increasing by $\sim 200 \%$ ( $\mathrm{p}<0.01$ ). The cellular apoptosis was further increased by $\sim 56.92 \%$ in the EGCG+UVA $(31.48 \pm 2.01)$ compared to the UVA only group $(\mathrm{p}<0.05)$, which implied that EGCG prompts apotosis of UVA damaged HSFs. In accordance with cell apoptosis, the percentage of cells in the cell cycle displayed a similar change; the number of cells in arrested S phase increased $(64.15 \pm 3.98 \%)$ and was accompanied by a decrease in cells in the G0/G1 (17.95 $\pm 1.47 \%)$ and G2/M phase $(17.9 \pm 1.21 \%)$. Compared to the UVA group, co-culture with EGCG reduced the number of cells in the $S$ phase $(26.78 \pm 1.10 \%$, $\mathrm{p}<0.001)$, and an increase in cells in the G0/G1 (2.7-fold) and G2/M phase (1.43-fold) $(p<0.05$, Table II) was noted. These results indicate that EGCG confers a photo-protective effect on UVA-irradiated HSFs. 
Table II. Effects of UV irradiation and EGCG treatment on apoptosis and the cell cycle in HSF.

\begin{tabular}{lcccc}
\hline Groups & Apoptosis $(\%)$ & S phase $(\%)$ & G0/G1 phase $(\%)$ & G2/M phase $(\%)$ \\
\hline Control & $0.15 \pm 0.06$ & $29.73 \pm 1.52$ & $46.50 \pm 2.32$ & $23.77 \pm 1.21$ \\
EGCG & $0.50 \pm 0.11$ & $30.36 \pm 1.83$ & $48.79 \pm 2.42$ & $20.85 \pm 1.05$ \\
UVA & $20.06 \pm 1.14$ & $64.15 \pm 3.98$ & $17.95 \pm 1.47$ & $17.90 \pm 1.21$ \\
UVA+EGCG & $31.48 \pm 2.01^{\mathrm{a}}$ & $25.78 \pm 1.10^{\mathrm{b}}$ & $48.59 \pm 2.68$ & $25.63 \pm 1.92$ \\
\hline
\end{tabular}

The conditioned HSFs were managed for apoptosis and cell cycle detection as above. Data are shown as the means \pm SD of three independent experiments. ${ }^{a}$ Pre-treatment with EGCG increased the cellular apoptosis compared to the UVA group (p<0.05). ${ }^{b}$ Pre-treatment with EGCG decreased the percentage of S phase cells compared to the UVA group $(\mathrm{p}<0.001)$.

\section{Discussion}

Solar UV irradiation induces different hazardous effects in the skin, including sunburn (18), photoaging (19) and cancer (20). Protection against solar-induced damage is therefore a highly desirable goal. Several studies have shown that EGCG has a photo-protective effect on human skin cells, such as primary keratinocytes, fibroblasts, dendritic cells, Langerhans cells and $\mathrm{HaCaT}$ cells (21-26). These data suggest that EGCG may protect HSFs against UVA-induced HPRT mutations in vitro.

It has been well known that DNA lesions are induced by UV irradiation and the mutations can be formed in some critical genes, which are believed to play an important role in carcinogenesis $(27,28)$. Previously, researchers have used the alkaline comet assay to compare the DNA damage induced by UVR in cultured human cells (lung fibroblasts, skin fibroblasts and epidermal keratinocytes) with and without EGCG, and found that EGCG protects human cellular DNA from UV and visible radiation-induced damage (29). In the present study, we confirmed that 2 weeks of UVA irradiation increased the mutation frequency of the HPRT gene approximately 150-240 times higher than that of the control and EGCG groups (intrinsic mutant levels). Pre-treatment with EGCG inhibited the frequency of mutation at the coding region of the HPRT gene by $47.85 \%$ in UVA-irradiated HSFs. Therefore, we may infer that one important photo-protective effect of EGCG on HSFs from multiple UVA irradiation may be related to the depression of DNA mutation formation.

Compared to freshly isolated HSF cells, the HSF cells that were cultured for more than 20 passages, with their special aging morphology, were positive for SA- $\beta$-Gal staining. However, upon pre-treatment with EGCG, not only the aging morphology was normalized, but also the number of SA- $\beta$ Gal-positive cells was markedly reduced, which indicated that EGCG mitigated the intrinsic senescence in cultured HSFs. However, after pre-treatment with EGCG with irradiation of UVA for a continuous 2 weeks, the positive number of senescent cells increased by $38.92 \%$ compared to the UVA group. This indicated that treatment of HSFs with EGCG prior to UVA exposure caused a further increase in the rate of senescence. Similarly, UVA also caused an increase in cell growth arrest and apoptosis rates, which were further increased by treatment of EGCG prior to UVA exposure.

Several studies have discussed the relationship between senescence and tumor genesis. Some researchers consider senescence as an important defence mechanism by preventing damaged or abnormal cells from cancer transformation $(12-14,30)$. On the one hand, by altering the tissue microenvironment, senescent cells may contribute to the rise in cancer that occurs with age. On the other hand, senescence is thought to be a powerful, albeit imperfect, tumor-suppressive mechanism since one of the phenotypic changes in senescent cells is irreversible growth arrest. Cellular apoptosis is also an effective mechanism for eliminating senescent or mutated cells to balance the intrinsic environment and interfere with tumor growth. Certain studies have confirmed that UV-induced cell apoptosis is also a protective mechanism in UV injury (31-33). Treatment of HSFs with EGCG prior to UVA exposure caused a further increase in the rates of senescence and apoptosis, but a decrease in the cell proliferation rate and mutant frequency. Thus, we may infer that a novel mechanism was initiated, in which the mutant HSFs induced by multiple UVA exposure underwent a state of arrested growth and entered senescence and/or a course of apoptosis.

Previous studies have shown that the WRN helicase may play a role in cancer development, by participating in both oncogenic proliferation through the avoidance of senescence, as well as protection of the genome from mutations that eventually promote tumor establishment (34). This is a paradigm that has been reported for telomerase as well. Similarly, activation of telomerase promotes cell immortalization and thus tumor genesis, while its absence protects cancer-prone mice from tumor development $(35,36)$. However, the exacerbated genomic instability, occurring after several generations in telomerase-negative mice, eventually contributes to an increased incidence of cancers (37). Accordingly, future studies are also required to assess the effect of EGCG on WRN and telomerase as an adaptive response for its efficacy against UVA-caused HPRT mutations in HSFs.

In summary, the results obtained in the present study indicate that EGCG induces multiple UVA damaged cells to proceed to either senescence and/or apoptosis. In this way, the mutant cells cannot be replicated and inherited, and photocarcinogenesis can be finally reduced. However, the exact molecular mechanisms remain unclear. Present and further studies concerning EGCG may certainly provide new clues for new strategies to avoid UV-induced damages.

\section{Acknowledgements}

This study was supported by grants from the China National Natural Science Foundation (30771946, 81000700). 


\section{References}

1. Wang SQ, Setlow R, Benrick M, et al: Ultraviolet A and melanoma: a review. J Am Acad Dermatol 44: 837-846, 2001.

2. Kappes UP, Luo D, Potter M, et al: Short- and long-wave UV light (UVB and UVA) induce similar mutations in human skin cells. J Invest Dermatol 126: 667-675, 2006.

3. Bickers DR and Athar M: Novel approaches to chemoprevention of skin cancer. J Dermatol 27: 691-695, 2000.

4. Bode AM and Dong Z: Signal transduction pathways: targets for chemoprevention of skin cancer. Lancet Oncol 1: 181-188, 2000

5. Zhou BR, Luo D, Lin XF, et al: Protective effect of the Baicalin against DNA damage induced by ultraviolet $B$ irradiation to mouse epidermis. Photodermatol Photoimmunol Photomed 24: $175-182,2008$

6. Morley N, Clifford T, Salter L, et al: The green tea polyphenol (-)-epigallocatechin gallate and green tea can protect human cellular DNA from ultraviolet and visible radiation-induced damage. Photodermatol Photoimmunol Photomed 21: 15-22, 2005 .

7. Katiyar SK: Skin photoprotection by green tea: antioxidant and immunomodulatory effects. Curr Drug Targets Immune Endocr Metabol Disord 3: 234-242, 2003.

8. Conney AH, Lou YR, Xie JG, et al: Some perspectives on dietary inhibition of carcinogenesis: studies with curcumin and tea. Proc Soc Exp Biol Med 216: 234-245, 1997.

9. Luo D, Min W, Lin XF, et al: Effect of epigallocatechin-gallate on ultraviolet B-induced photo-damage in keratinocyte cell line. Am J Chin Med 34: 911-922, 2006.

10. Hirai Y, Kusunoki Y, Kyolzumi S, et al: Mutant frequency at the HPRT locus in peripheral blood T lymphocytes of atomic bomb survivors. Mutat Res 329: 183-196, 1995.

11. Albertini RJ: HPRT mutations in human: biomarkers for mechanistic studies. Mutat Res 489: 1-16, 2001.

12. Campisi J: The role of cellular senescence in skin aging. J Investig Dermatol Symp Proc 3: 1-5, 1998

13. Shay JW and Roninson IB: Hallmarks of senescence in carcinogenesis and cancer therapy. Oncogene 23: 2919-2933, 2004.

14. Campisi J: Suppressing cancer: the importance of being senescent. Science 309: 886-887, 2005.

15. Huang C, Ma WY, Goranson A, et al: Resveratrol suppresses cell transformation and induces apoptosis through p53-dependent pathway. Carcinogenesis 20: 237-242, 1999.

16. Pendergrass WR, Lane MA, Bodkin NL, et al: Cellular proliferation potential during aging and caloric restriction in rhesus monkeys. J Cell Physiol 180: 123-130, 1999.

17. Dimri GP, Lee X, Basile G, et al: A biomarker that identifies senescent human cells in culture and in aging skin in vivo. Proc Natl Acad Sci USA 92: 9363-9367, 1995.

18. Honigsmann H: Erythema and pigmentation. Photodermatol Photoimmunol Photomed 18: 75-81, 2002.

19. Fisher GJ, Kang S, Varani J, et al: Mechanisms of photoaging and chronological skin aging. Arch Dermatol 138: 1462-1470, 2002.

20. Gruijl FR, Kranen HJ and Mullenders LH: UV induced DNA damage, repair, mutations and oncogenic pathways in skin cancer. J Photochem Photobiol B 63: 19-27, 2001.
21. Ji X, Luo D, Lin XF, et al: The phosphorylated P53 protein expressions in epidermal Langerhans cells irradiated by UVB. J Clin Dermatol 36: 134-136, 2007.

22. Ji X, Luo D, Miao X, et al: Inhibitory effect of EGCG on apoptosis of Langerhans cells after UVB irradiation. Chin J Dermatol 39 344-346, 2006

23. Luo D, Xu J, Zhou BR, et al: Influence of EGCG in different formulations on the apoptosis of epidermis cells of $\mathrm{Balb} / \mathrm{C}$ mice irradiated by UVB. J Chin Pharmaceut Univ 38: 535-538, 2007.

24. Luo D, Zhou BR and Ji X: Influence of epigallocatechin gallate on the immune function of dendritic cells after ultraviolet $\mathrm{B}$ irradiation. J Microbiol Immunol 5: 90-98, 2007.

25. Luo D, Lin XF, Xu J, et al: Effects and regulatory mechanisms of three traditional Chinese medicines on HACaT cells irradiated by UVB. Chin Pharmacol Bull 23: 750-755, 2007.

26. Li M, Luo D and Lin XF: Photoprotection of EGCG on HaCaT cells against oxidative damage and apoptosis from UVA irradiation. J Clin Derm 37: 80-83, 2008.

27. Wang YC, Maher VM, Mitchell DL, et al: Evidence from mutation spectra that the UV hypermutability of xeroderma pigmentosum variant cells reflects abnormal, error-prone replication on a template containing photoproducts. Mol Cell Biol 13: 4276-4283, 1993

28. Xu G, Snellman E, Bykov VJ, et al: Effect of age on the formation and repair of UV photoproducts in human skin in situ. Mutat Res 459: 195-202, 2000

29. Salter L, Clifford T, Morley N, et al: The use of comet assay data with a simple reaction mechanism to evaluate the relative effectiveness of free radical scavenging by quercetin, epigallocatechin gallate and N-acetylcysteine in UV-irradiated MRC5 lung fibroblasts. J Photochem Photobiol B 75: 57-61, 2004.

30. Beausejour CM, Krtolica A, Galimi F, et al: Reversal of human cellular senescence: roles of the p53 and P16 pathways. EMBO J 22: 4212-4222, 2003

31. Neades L, Cox J and Pelling O: S-phase arrest in mouse keratinocytes exposed to multiple doses of ultraviolet B/A radiation. Mol Carcinog 23: 159-167, 1998.

32. Li G and Ho VC: p53-dependent DNA repair and apoptosis respond differently to high- and low-dose ultraviolet radiation. Br J Dermatol 139: 3-10, 1998.

33. Chow $\mathbf{J}$ and Tron VA: Molecular aspects of ultraviolet radiationinduced apoptosis in the skin. J Cutan Med Surg 9: 289-295, 2005.

34. Grandori C, Wu KJ, Fernandez P, et al: Werner syndrome protein limits MYC-induced cellular senescence. Genes Dev 17: 1569-1574, 2003

35. Greenberg RA, Chin L, Femino A, et al: Short dysfunctional telomeres impair tumorigenesis in the INK4a(delta2/3) cancerprone mouse. Cell 97: 515-525, 1999.

36. Blasco MA: Telomeres in cancer and aging: lessons from the mouse. Cancer Lett 194: 183-188, 2003.

37. Rudolph KL, Chang S, Lee HW, et al: Longevity, stress response, and cancer in aging telomerase-deficient mice. Cell 96: 701-712, 1999. 